\title{
Path-Following Control based on Ground-Watching Navigation
}

\author{
Qiheng Miao and David Cebon*
}

\begin{abstract}
This paper discusses the path-following performance of actively-steered articulated vehicles based on measurement signals from two 'ground-watching' navigation systems (GWNS). The ground-watching navigation systems are described. These use high-speed USB-3 cameras and an image processing computer to measure and calculate off-tracking distance for a path-following steering controller. Full-scale field tests are performed using an experimental articulated vehicle, tested under both open-loop and closed-loop conditions. Off-tracking distance at the rear camera with respect to the front camera is controlled to be less than $0.1 \mathrm{~m}$ for tests with both GWNSs.
\end{abstract}

Index Terms - path following; active steering; articulated vehicles; ground-watching navigation

\section{INTRODUCTION}

$I_{8}$ this paper, a path-following trailer steering system based on ground-watching navigation is investigated for low adhesion, non-level, 'off-highway' conditions. Such conditions are typically seen in freight applications such as: milk/livestock collections from farms, transportation of raw materials for primary industries such as logging and sugarcane and military supply.

The application of high accuracy path-following trailer steering in these transport operations would relax the low-speed manoeuvrability constraints that limit the capacity of the existing vehicles [1]. It could enable use of tractor-semitrailer vehicles instead of short rigid lorries for some farm collections, and use of multiple-unit articulated vehicles instead of tractor-semitrailers for logging, sugarcane and military supply.

By using higher capacity vehicles in these operations, fuel consumption (greenhouse gas emissions) and shipping costs could be reduced significantly. In Canada, shipping costs, emissions and traffic congestions were reduced by $20-30 \%$ when long combination vehicles were introduced in the $1980 \mathrm{~s}$ [2]. For transport of military supplies, fewer trips and fewer drivers would also ensure less exposure to military threat, improving the safety of the supply line.

The path-following controller investigated in this study was designed to minimize the 'lateral off-tracking' error (illustrated

This paper is submitted on August, 2016. The work included in this paper was supported by the Cambridge Vehicle Dynamics Consortium (CVDC).

Qiheng Miao is with the Department of Engineering, Cambridge University, Cambridge, CB2 1PZ, UK (e-mail: qm206@ cam.ac.uk).

David Cebon is with the Department of Engineering, Cambridge University, Cambridge, CB2 1PZ, UK (corresponding author, e-mail: dc@ eng.cam.ac.uk). as $\Delta y$ in Fig.1 (a)) at the 'follow point' (middle of the rear doors of the trailer) relative to the 'lead point' (5th-wheel hitch). Its performance was found to degrade under adverse conditions (such as road camber and grade combined with low-adhesion) due to inaccurate off-tracking estimation caused by the errors in estimated sideslip and measured velocity [3].

Good path-following would be achieved under such conditions if lateral off-tracking could be estimated or measured accurately. A navigation system is therefore needed to provide reliable position data under such conditions. In the development of off-highway navigation systems for articulated vehicles, much can be gained by studying existing vehicle navigation systems [4]. The goal of navigation systems for active steering is to measure lateral and longitudinal wheel-slip or accurate position information for various path-following strategies. The conventional approaches generally include the use of the Global Positioning System (GPS), inertial navigation systems (INS) or vision systems.

A review of literature [5] indicated a number of drawbacks and practical constraints of using GPS or GPS/INS integrations for off-highway vehicle position/heading measurement. Civil GPS can only provide position measurement within the accuracy of a few meters and has a low update rate of 1 to $10 \mathrm{~Hz}$ [6]. Its integration with INS could yield more accurate position measurements but would cost over 30,000 GBP (RT3000 as an example). The use of state estimators based on vehicle dynamics was also found to be unsuitable off-highway, due to complex vehicle dynamics and unreliable sensor measurements. The use of image data was investigated and found to be promising for a variety of operating conditions. Hence, vision systems are further investigated in this paper to fulfil the goal of navigation off-highway.

Automated vehicle guidance systems typically follow a desired path by recognising landmarks on the road, e.g. a white line along the centre or edge of the road. In image data, these stationary landmarks can be represented and matched as a pattern of pixel intensities [7,8] or a series of handcrafted feature combination such as corners or edges [9, 10]. The former representation is denser and more robust to low-texture environment; while the later representation is sparser and invariant to illumination changes. By sequentially localizing the vehicle relative to such landmarks, ego-motion of the vehicle can be deduced from image or video sequences. Similar ideas are adopted by the concept of ground-watching navigation (see Section 2). 

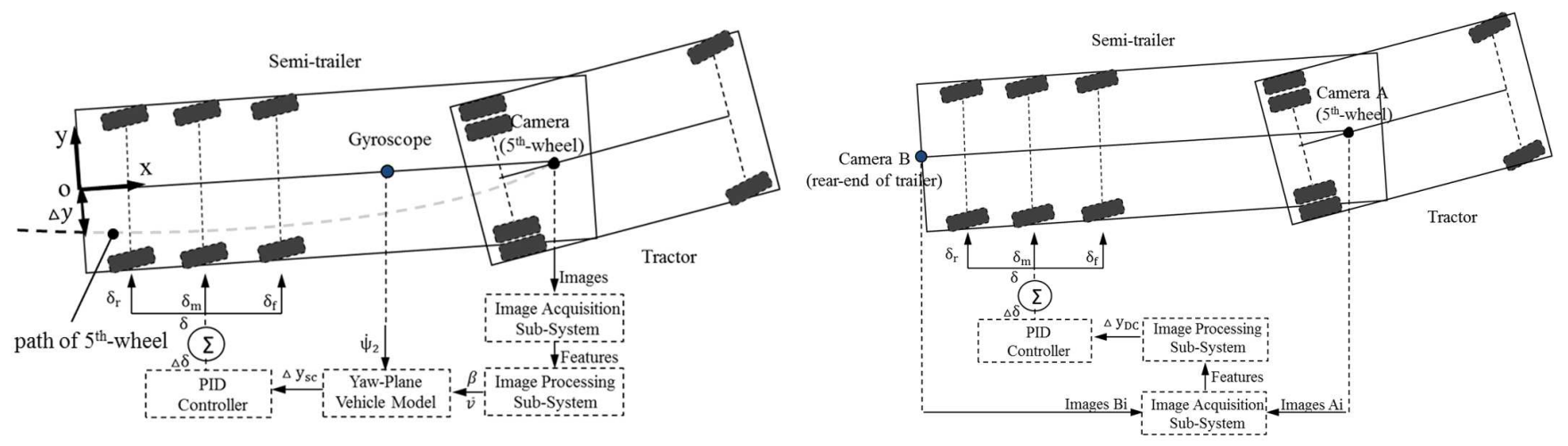

(a) single-camera ground-watching navigation system (SC-GWNS)

Fig. 1. Schematics of the two ground-watching navigation systems (GWNSs)

(b) dual-camera ground-watching navigation system (DC-GWNS)

The main contributions of this paper are: (i). development of vision-based navigation for trailer steering applications; (ii) modification of trailer steering control to handle steering saturation; (iii) full-scale field testing and verification of the closed-loop system.

\section{GROUND-WATCHING NAVIGATION SYSTEMS}

Two ground-watching navigation systems (GWNS) were developed to provide the necessary vehicle states using cameras. They capture and analyse images of the road surfaces along the path travelled by the vehicle. One GWNS has a single camera mounted at the lead point (tractor 5th wheel). The other has two cameras, the first camera mounted at the lead point and the second mounted at the follow (trailer rear end) point. They share the same image processing pipeline: feature extraction, description, matching and then camera motion recovery from the matched features. However, they differentiate in image input and system output.

\section{A. Single-Camera Ground-Watching Navigation System}

The single-camera ground-watching navigation system (SC-GWNS) aims to measure accurately both absolute speed, $|v|$, and sideslip, $\beta$, at the lead point (tractor 5th-wheel) of the steering controller. These signals are then fed into a yaw-plane vehicle model together with yaw rate measurements, $\dot{\psi}_{2}$, from a gyroscope on the trailer unit. Positions of the $5^{\text {th }}$ wheel can thus be calculated and saved in a data buffer. The path of the $5^{\text {th }}$ wheel is then transformed into a trailer-based coordinate (see Fig. 1 (a)), yielding lateral off-tracking error $\Delta y$. This is passed to the steering controller, and the demanded trailer steer angles are generated. The working principle of the SC-GWNS is illustrated in Fig. 1(a).

This system can provide accurate off-tracking signals for a wide working range of off-tracking errors (up to a few metres). However, the accuracy of this system is subject to errors due to drift in yaw rate integration. The off-tracking errors would be within centimetres if the bias in yaw rate was carefully removed [5].

\section{B. Dual-Camera Ground-Watching Navigation System}

Unlike the SC-GWNS, the dual-camera ground-watching navigation system (DC-GWNS) directly determines lateral off-tracking distance by comparing images collected at the lead and follow points, and thus determines the necessary trailer steer angles. Road features from the lead point camera are saved in the data buffer (instead of the position along the path of the $5^{\text {th }}$ wheel). By matching the features captured at the rear end of the trailer to features saved in the buffer, lateral off-tracking distance can be measured. The working principle of the DC-GWNS is illustrated in Fig.1(b).

Compared to the SC-GWNS, this system is not subject to the bias in yaw rate signals. It can measure off-tracking distance with errors smaller than $1 \mathrm{~cm}$. However, the working range of this system is limited to lateral off-tracking of $0.3 \mathrm{~m}-0.5 \mathrm{~m}$ in order to ensure that the paths of both cameras overlap sufficiently [5].

Although using an extra camera would increase the cost of the overall system slightly, the accuracy gained for off-tracking measurement would be of great benefit when precise path-following is required, eg in traversing partially-cleared mine fields or operating on slippery mountain roads.

\section{STEERING CONTROLLER}

Cheng's path-following steering controller [11] was found unable to follow the desired path on cambered or slippery roads due to unmeasured longitudinal and lateral slip of the tyres [3]. Moreover, Cheng's controller used measurements from both the tractor (front steer angle and wheel speed) and the trailer (articulation angle and yaw rate). Therefore, some 'tuning' is needed to adjust the parameters when such controllers are implemented with different tractor units. An entirely trailer-based controller would eliminate these efforts, making it easier to operate such trailers with different tractors. This is important due to the modularity with which articulated HGVs are normally operated.

The GWNSs introduced in this paper are entirely trailer-based. They are used to replace the articulation angle sensor, steer angle sensor and the wheel speed sensors. Trailers can be actively steered using the measured data. 


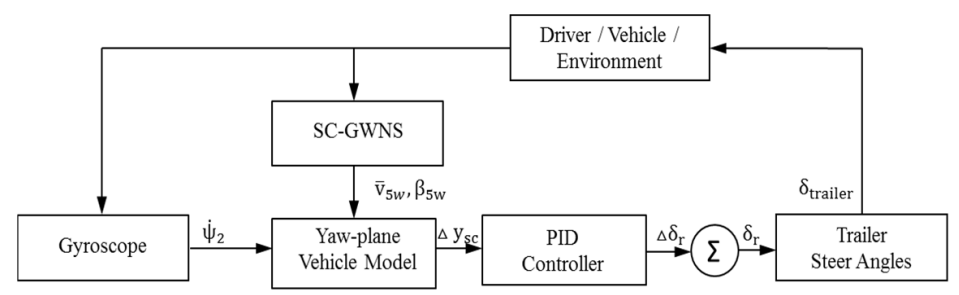

(a) single-camera ground-watching navigation system (SC-GWNS) Fig. 2. Schematic of the path-following control based on GWNSs.

For the SC-GWNS, sideslip and forward speed of the trailer are measured near the 5th-wheel and fed into a yaw-plane vehicle model with the measured yaw rate (see Fig. 2(a)). The signals are used to calculate the lateral off-tracking at the follow point. The distance travelled down the path by the 5 thwheel of the tractor can be calculated by:

$$
S_{5 w}=\int v_{5 w} d t
$$

Unlike Cheng's work [11], the position data of lead and follow points are calculated using trailer states:

$$
\begin{gathered}
\gamma_{5 \mathrm{w}}=\psi_{2}+\beta_{2}^{5 \mathrm{w}} \\
\mathrm{X}_{5 \mathrm{w}}=\int \mathrm{v}_{5 \mathrm{w}} \cos \left(\gamma_{5 \mathrm{w}}\right) \mathrm{dt} \\
\mathrm{Y}_{5 \mathrm{w}}=\int \mathrm{v}_{5 \mathrm{w}} \sin \left(\gamma_{5 \mathrm{w}}\right) \mathrm{dt} \\
\mathrm{X}_{\mathrm{r}}=\mathrm{X}_{5 \mathrm{w}}-\mathrm{L} \cos \left(\Psi_{2}\right) \\
\mathrm{Y}_{\mathrm{r}}=\mathrm{Y}_{5 \mathrm{w}}-\mathrm{L} \sin \left(\Psi_{2}\right)
\end{gathered}
$$

Where: $\mathrm{X}_{5 \mathrm{w}}, \mathrm{Y}_{5 \mathrm{w}}, \mathrm{X}_{\mathrm{r}}, \mathrm{Y}_{\mathrm{r}}=\mathrm{x}$ and $\mathrm{y}$ position of tractor $5^{\text {th }}$ wheel and trailer rear end in global coordinates [m] $\psi_{2}=$ yaw angle of trailer unit [rad] $\gamma_{5 \mathrm{w}}=$ heading angle of tractor $5^{\text {th }}$ wheel [rad] $\mathrm{v}_{5 \mathrm{w}}=$ absolute speed of tractor $5^{\text {th }}$ wheel $[\mathrm{m} / \mathrm{s}]$ $\beta_{2}^{5 \mathrm{w}}=$ sideslip angle of tractor $5^{\text {th }}$ wheel [rad] $\mathrm{L}=$ length of trailer unit $[\mathrm{m}]$

For the DC-GWNS, the lateral off-tracking is measured directly and passed to the controller (see Fig.2(b)). A Proportional-Integral-Derivative (PID) controller is then employed to minimise the off-tracking by generating a steering angle for a 'virtual driver' at the follow point [7]:

$$
\begin{gathered}
\Delta \delta_{\mathrm{v}}=\mathrm{K}_{\mathrm{PID}} \Delta \mathrm{e}_{\mathrm{v}} \\
\delta_{\mathrm{v}}=\delta_{\mathrm{v} 0}+\Delta \delta_{\mathrm{v}}
\end{gathered}
$$

Where: $\delta_{\mathrm{v}}, \delta_{\mathrm{v} 0}=$ steer angle of virtual wheel at current and last time step [rad]

$\Delta \mathrm{e}_{\mathrm{v}}=$ change of path-tracking errors [m]

$\Delta \delta_{\mathrm{v}}=$ change of steer angle of virtual wheel after a sample time [rad]

If a controller with fixed time step is used, the position data can be stored in a data buffer on each time step for each GWNS. However, when the vehicle is travelling very slowly, the data buffer would become very large. An alternative approach is to

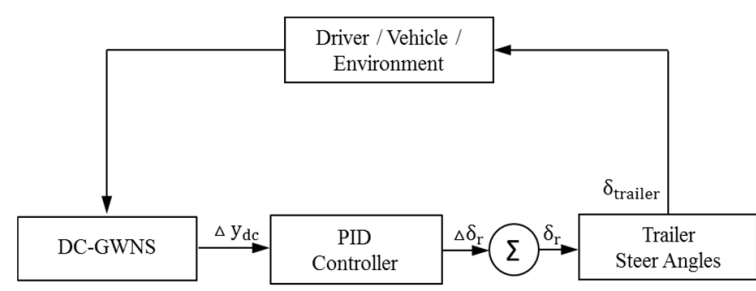

(b) dual-camera ground-watching navigation system (DC-GWNS)

store the path data at a set number of points with fixed distance spacing along the length of the trailer [5]. The 5th wheel position at a particular time can be interpolated linearly from this array. The spacing was set to be $0.1 \mathrm{~m}$ with a buffer size of 200 in the simulation. These settings ensured that a sufficiently long path could be stored in the buffer at appropriate resolution.

The steer angle from the virtual driver is limited to be less than $90^{\circ}$ in the controller. This ensures that its tangent value is monotonic in the limited range. The steer angles of all trailer axles can be determined from the geometry of the vehicle, by assuming zero sideslip of the tyres:

$$
\delta_{\mathrm{i}}=\tan ^{-1}\left(\frac{\mathrm{l}_{\mathrm{ir}}}{\mathrm{l}_{\mathrm{i}}} \tan \left(\beta_{\mathrm{f}, \mathrm{i}}\right)+\frac{\mathrm{l}_{\mathrm{if}}}{\mathrm{l}_{\mathrm{i}}} \tan \left(\delta_{\mathrm{v}, \mathrm{i}}\right)\right)
$$

Where: $\quad l_{i}=$ distance between the lead and the follow points on trailer ' $i$ ' [m] $l_{\text {if }}=$ distance between the axle and the lead point on trailer ' $\mathrm{i}$ ' $[\mathrm{m}]$

$l_{\text {ir }}=$ distance between the axle and the follow point on trailer 'i' $[\mathrm{m}]$

$\beta_{\mathrm{f}, \mathrm{i}}=$ sideslip angle at the lead point on trailer ' $\mathrm{i}$ ' [rad] $\delta_{\mathrm{v}, \mathrm{i}}=$ steer angle of virtual wheel at the follow point on trailer 'i' [rad]

Trailer steer angles may reach actuator limits in very tight corners. During this period of saturation, the integral action in the PID controller will continue to integrate the errors and therefore produce erroneous control signals. This can cause undesirable effects such as excessive overshoot and subsequent oscillation when saturation ends.

A modified feedback approach [12] was employed in the controller to compensate for the 'wind-up' effects caused by saturation. It recomputed the integral term in the controller when the output saturated (see Fig. 3). From the figure, the system has an extra feedback path that forms an error signal from the difference between the controller output and the

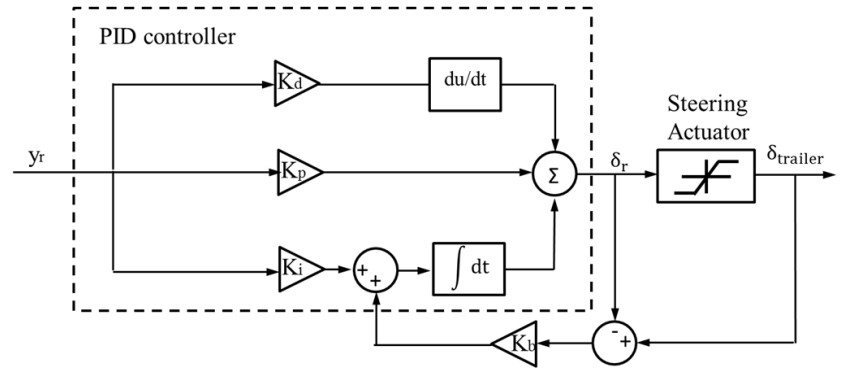

Fig. 3. Schematic of the steering controller based on GWNSs. 


\begin{tabular}{lccc}
\hline Sensor & Measurement & $\begin{array}{c}\text { Mean } \\
\text { Error }\end{array}$ & $\begin{array}{c}\text { Standard } \\
\text { Deviation }\end{array}$ \\
\hline SC-GWNS & Sideslip [degree] & 0.02 & 0.4 \\
& Absolute Velocity [m/s] & 0.002 & 0.023 \\
DC-GWNS & Off-Tracking [mm] & 2 & 2.3 \\
Yaw Gyro & Yaw rate [rad/s] & 0.02 & 0.003 \\
\hline
\end{tabular}

Table 1: Measurement noise of the GWNSs

actuator output. This error signal, which is zero if there is no saturation, is then fed to the input of the integrator through a gain, $\mathrm{K}_{\mathrm{b}}$. When saturation occurs, the integrator is driven to minimise the modified integral input. The control output will settle marginally outside the saturation limit. This ensures that the integrator reacts rapidly to the changes in error signal, therefore avoiding winding up.

\section{SimUlation STUDY}

\section{A. Simulation Modelling}

A 6-DoF tractor and semi-trailer model, developed in [3] was employed to simulate vehicle performance with the GWNSs. The model consists of five sub-models that interact with each other. For a given manoeuvre, the drive torque and steer angle of the tractor unit are determined by the driver model. The trailer steering angles are determined by the trailer steering controller. In addition to the steer angles, the vertical load on each tyre is also required to calculate the lateral force on each tyre. This information is fed into the vehicle dynamics model, completing the loop; with the states of each vehicle unit determined and passed back to the other sub-models. For details of the vehicle model, please refer to [3].

A $450^{\circ} \mathrm{UK}$ standard roundabout (with $5.3 \mathrm{~m}$ inner radius, $12.5 \mathrm{~m}$ outer radius and straight tangential lead-in and run-out paths [1]) was simulated on a road with a $5^{\circ}$ road camber. The noise of GWNSs was modelled as band-limited white noise. The mean and standard deviations of the noise signal are given in Table 1. Bias in yaw rate gyro measurements was assumed to have been perfectly compensated in the simulations.

The Ziegler-Nichols method [13] was used to tune the PID gains. This is a heuristic method which initially determined the control gain, Kc, and the oscillation period, Tc, of a proportional-only control by trial and error. The subsequent proportional, integral and derivative gains were determined by a rule of thumb for a classic PID controller [13]:

$$
\begin{aligned}
\mathrm{K}_{\mathrm{P}} & =0.6 \mathrm{~K}_{\mathrm{c}} \\
\mathrm{K}_{\mathrm{I}} & =\frac{2 \mathrm{~K}_{\mathrm{p}}}{\mathrm{T}_{\mathrm{c}}} \\
\mathrm{K}_{\mathrm{D}} & =\frac{\mathrm{K}_{\mathrm{P}} \mathrm{T}_{\mathrm{c}}}{8}
\end{aligned}
$$

The resulting controllers with a proportional gain of 0.9 , an integral gain of 4.0 and a derivative gain of 0.05 were used in the simulations. Both navigation systems were set to operate at a frame-rate of $10 \mathrm{~Hz}$ in the simulations.

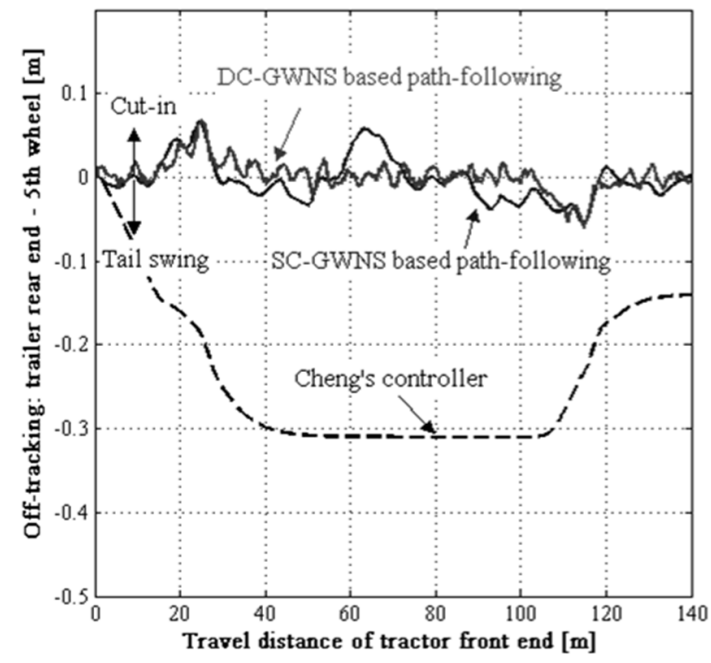

(a) with no initial off-tracking

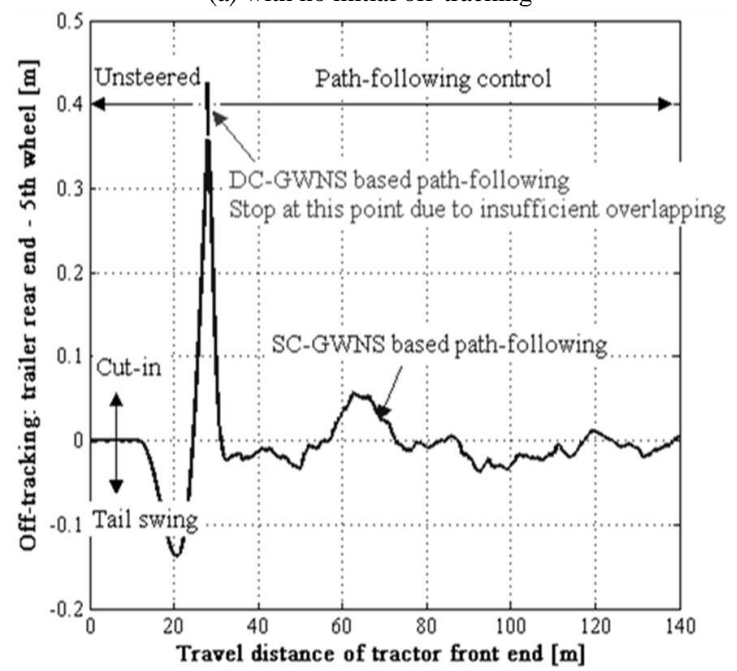

(b) with initial off-tracking

Fig. 4: Path-following performance of the SC-GWNS and the DC-GWNS for the $450^{\circ} \mathrm{UK}$ standard roundabout manoeuvre

\section{B. Simulation Results}

Fig. 4(a) shows the path-following performance when initial off-tracking was set to be zero in the simulation for the $450^{\circ}$ UK standard roundabout manoeuvre. This means both GWNSs and the corresponding path-following control can work throughout the entire roundabout manoeuvre. In the figure, lateral off-tracking of path-following control based on SC-GWNS is presented as the red line while the counterpart using DC-GWNS is shown as the blue line. Compared to Cheng's controller, both navigation systems performed better path-following in the simulation, showing no steady-state off-tracking errors on average. The transient path-following responses are similar for both systems. However, the DC-GWNS outperforms the SC-GWNS in steady-state performance, exhibiting less oscillation in off-tracking errors.

Since the simulation in Fig. 4(a) was initialised with no lateral off-tracking, using either of the GWNSs would ensure that rear end of the trailer followed the path of tractor 5th-wheel. However, if off-tracking exceeds $300 \mathrm{~mm}$, insufficient overlapping of the image data for the DC-GWNS can occur and 

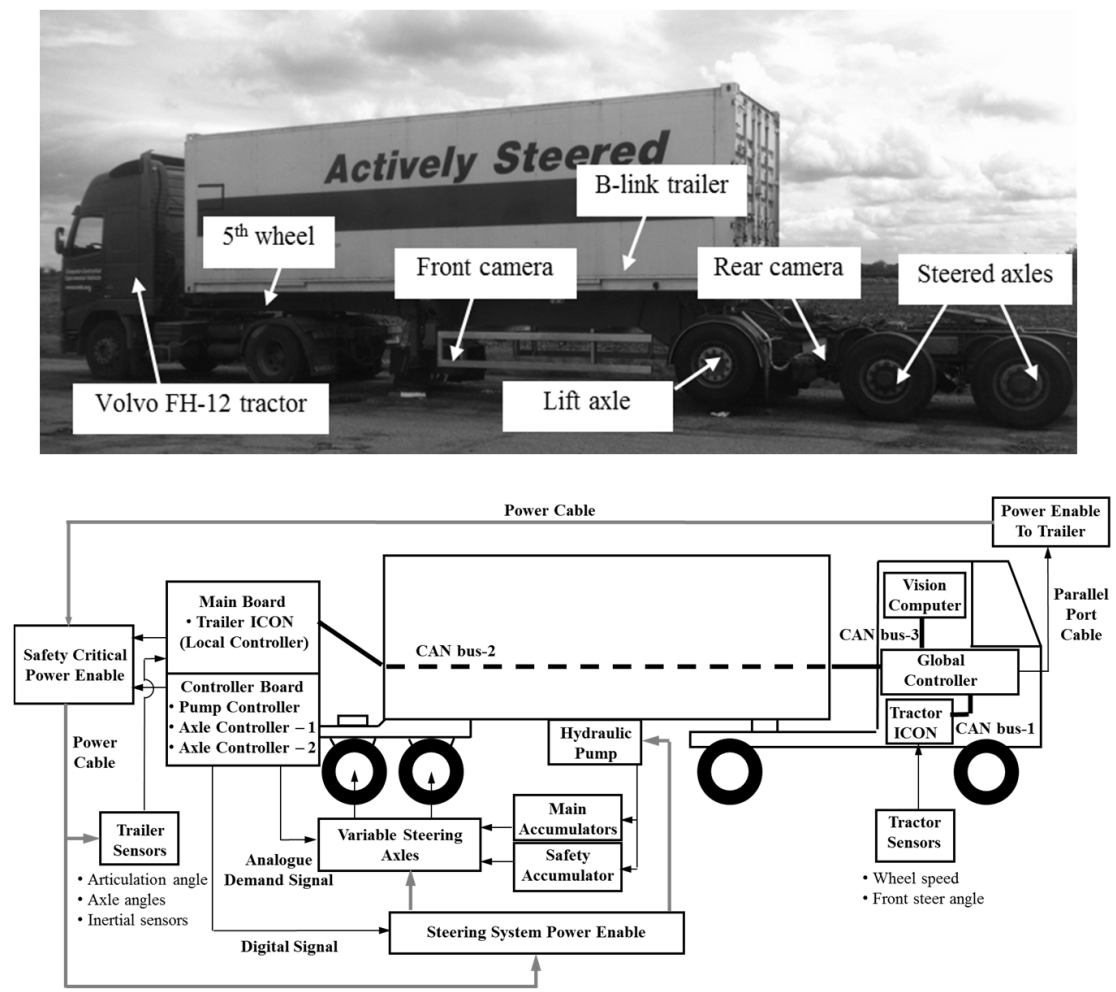

Fig. 5. CVDC active steering experimental vehicle

the DC-GWNS can fail, preventing further steering control. This can happen during a cornering manoeuvre if the vehicle loses vision - e.g. through poor image conditions such as passing a puddle or experiences saturation of trailer steer angles. In which case, the DC-GWNS cannot navigate the controller whereas the SC-GWNS can potentially steer the trailer back to the desired path.

The vehicle model was used to simulate the UK standard roundabout manoeuvre with trailer axles 'locked' to the centre for the first $9 \mathrm{~s}$ of the simulation. The consequent path-following performance is shown in Fig. 4(b). This resulted in lateral off-tracking of approximately $0.4 \mathrm{~m}$ at the rear end of the trailer by the time the vehicle had travelled $28 \mathrm{~m}$ along the path. Trailer steer angles were applied using measurements from the SC-GWNS from then on. From the figure, lateral off-tracking was controlled to around zero within $1 \mathrm{~s}$ after trailer steering was applied and remained within $0.1 \mathrm{~m}$ thereafter.

\section{FIELD TESTING}

\section{A. Testing Setup}

The CVDC experimental vehicle consisted of a 2-axle Volvo FH-12 tractor and a 3-axle 'link' trailer unit (see Fig. 5). The tractor was fitted with a variety of sensors, including front steer angle, wheel speed and inertial sensors. The active-steering link-trailer is an $11 \mathrm{~m}$ long tri-axle trailer, designed for use in a B-double combination. The rear two axles are fitted with hydraulic steering actuators. The front axle was lifted off the road for these tests. The steering actuators were developed by 3D Evolution Ltd (3DE) according to specifications provided by the CVDC in a previous project. Each axle can be independently steered up to $30^{\circ}$, in response to the demanded steer angles.

The trailer was also fitted with a variety of sensors, including a 6-axis inertial sensor box, an articulation angle sensor, an RT3000 inertial and GPS navigation system and the GWNSs. Only the signals from the GWNSs and inertial sensor box were fed into the active steering controller. The other sensor signals were used for post-processing.

The GWNSs consist of high-speed digital cameras and a vision processing computer with USB-3 camera interfaces. A powerful 4-core PC with a clock speed of $3.6 \mathrm{GHz}$ and solid-state hard drive (SSD), running the Ubuntu 14 Linux operating system, was used as the vision processing computer. Two systems, i.e. the SC-GWNS and the DC-GWNS, were setup in parallel on the test vehicle.

A distributed, multi-level control system was used to control the trailer's steering system (see Fig. 5). It consists of a global controller (top level), two local controllers (intermediate level), the GWNSs, inertial sensors and actuator drive junction boxes (lowest level). The global controller interfaced with the vision processing computer and two local controllers via CANbus. It enabled the 24V DC power supply to power the trailer unit via a signal from its parallel port when the controller was started up. The vision processing computer processed image data and sent navigation data (speed, sideslip and off-tracking) to the global controller via the CANbus. The local controllers logged, digitised and filtered sensor signals from the sensors on both vehicle units and sent them to the global controller. The global controller passed control mode and demand trailer steer angle 


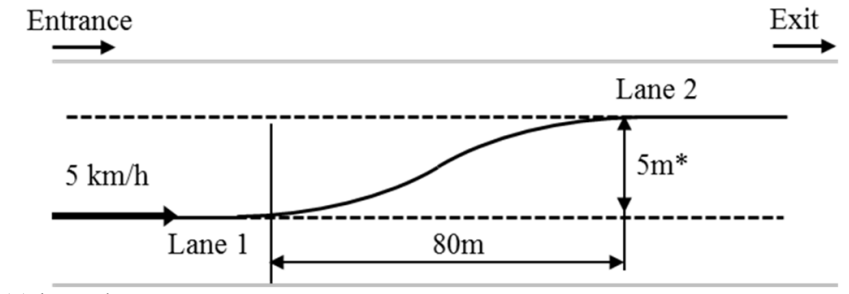

(a) lane-change manoeuvre

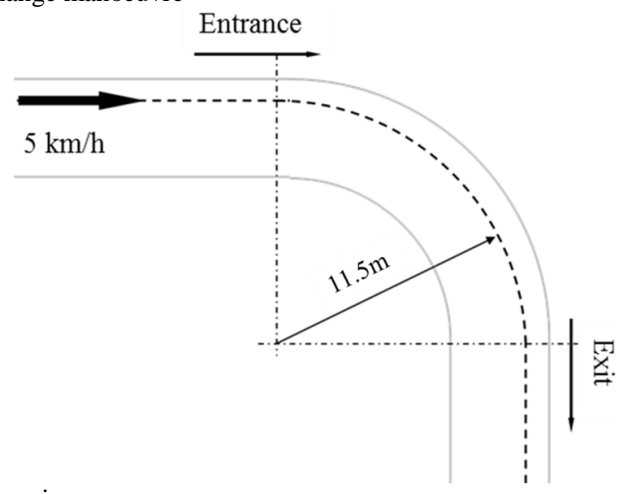

(b) $90^{\circ}$ cornering manoeuvre

Fig. 6. Illustration of testing manoeuvres

signals to the trailer local controller. Steer angles were applied on each trailer axle by the steering actuators.

In previous active steering projects digital cameras were used to measure off-tracking with high visibility temporary white lines painted on the test surface. This approach may potentially influence the performance of the GWNS since the paint would affect features in the image. In this project, the RT3000 inertial and GPS navigation system (RT3000) from Oxford Technical Solutions (OxTS) [14] was utilised to yield the 'ground truth' for off-tracking measurements. The accuracy of this system was verified by Rimmer [15] who found "that off-tracking errors measured by the RT3000 position sensor agreed with the 'line-tracking' cameras within approximately $0.1 \mathrm{~m}$ '. The RT3000 was placed on the floor of the trailer. Its signals were logged and transmitted via a CANbus cable which ran down the length of the vehicle.

\section{B. Testing Initialisation}

The intrinsic camera parameters of the GWNSs were calibrated before testing. The cameras were focused and aligned while the suspension was set to its normal height (the height when vehicle is moving). The first step of initialising the GWNSs was to align the geometric centres of cameras to the trailer central line. The next step was to eliminate the heading offset between the cameras and the trailer. The angular offset was measured in a straight-line manoeuvre and eliminated thereafter. The last step of GWNS initialisation was to calculate a scale factor to convert camera measurements into motion over the ground in metres. This was performed by driving the test vehicle in a straight-line for a known distance.

Every time the RT3000 is powered on, it goes through a calibration phase where the vehicle must first be driven in a straight-line above $5 \mathrm{~m} / \mathrm{s}$ and then it continues to calibrate itself upon further driving around (not necessarily in a straight line). The gain for trailer yaw rate signals was calibrated by rotating the sensor around its $\mathrm{z}$-axis through a known angle on a flat surface. The sensor offset was removed at the beginning of each test.

The steering controller gains derived from the simulation were tuned slightly by trial and error in the vehicle testing. This accounted for small differences between the simulation model and the experimental setup, due to simplifications in the model and constraints in the practical camera-installation on the test vehicle. Two sets of PI control gains were applied in the closed-loop tests for the input signals from different GWNSs. For the path-following steering controller using the signals from the SC-GWNS, a proportional gain of 0.5 and an integral gain of 0.6 were found to generate adequate performance. For the controller based on the DC-GWNS signals, a proportional gain of 0.1 and an integral gain of 0.12 were found to generate good performance.

A safety system for the active steering system was developed based on [16]. It was important not to damage the actuators through excessive input demands, particularly while commissioning the system. Sensor checks were performed for the demand and actual steer angle signals. If any of these signals exceeded a threshold, an emergency shutdown was performed. The threshold was tuned slightly differently for different manoeuvres during commissioning. The hardware of the active steering system and the safety system were initially tested using a 'sine-wave' trailer steering input before the closed-loop tests were performed.

\section{Testing Manoeuvres}

Field testing was performed at the vehicle speed of $5 \mathrm{~km} / \mathrm{h}$. Three manoeuvres were conducted: a straight-line, a lane change and a $90^{\circ}$ corner. The tests were conducted in two stages for the three manoeuvres. In the first stage, the global controller worked in the 'locked steering' mode and the GWNSs were tested in open-loop. In the second stage, the controller worked in the 'path-following' mode and the trailer axle was steered based on GWNS measurements with the lead and follow points set to the front and rear cameras. Each test was repeated twice.

The straight-line manoeuvre aimed to evaluate the basic operation of the GWNS-based path-following controller. This is the simplest manoeuvre for which the image data from both cameras should have the largest overlapping areas. Off-tracking distance was expected to be close to $0 \mathrm{~m}$ for this manoeuvre and the demand trailer steering angle should be around $0^{\circ}$ for the closed-loop test. This manoeuvre was performed by the test driver following the painted straight-line on the testing ground.

The lane change manoeuvre (see Fig.6(a)) started with a straight-line on one lane and ended with a straight-line on the other lane, turning smoothly in the transient region between the lanes. 
The $90^{\circ}$ cornering manoeuvre is shown in Fig. 6(b). The radius of the corner was $11.5 \mathrm{~m}$, which is the same as the UK standard roundabout. The test vehicle entered the corner after a straight-line and ended up with another straight-line after exiting the corner. Off-tracking distance at the rear camera with respect to the front camera was expected to exceed $1 \mathrm{~m}$ without trailer steering.

\section{Testing Results}

\section{1) Straight-line}

Open-loop testing results for the straight-line are shown in Fig. 7. From the figure, off-tracking measurements from the DC-GWNS agree well with the off-tracking signals from the SC-GWNS and the RT3000, with less than $0.05 \mathrm{~m}$ difference between these signals. It can also be seen from the figure that the off-tracking distance measured by the DC-GWNS (dark grey) has slightly smaller oscillation than the SC-GWNS calculation (light grey). This agrees with the findings from the simulation. The difference between the off-tracking distance signals from the two GWNSs in the tests is less obvious than predicted by the simulation. This is mainly because the distance between the cameras $(5.45 \mathrm{~m})$ is smaller than the distance

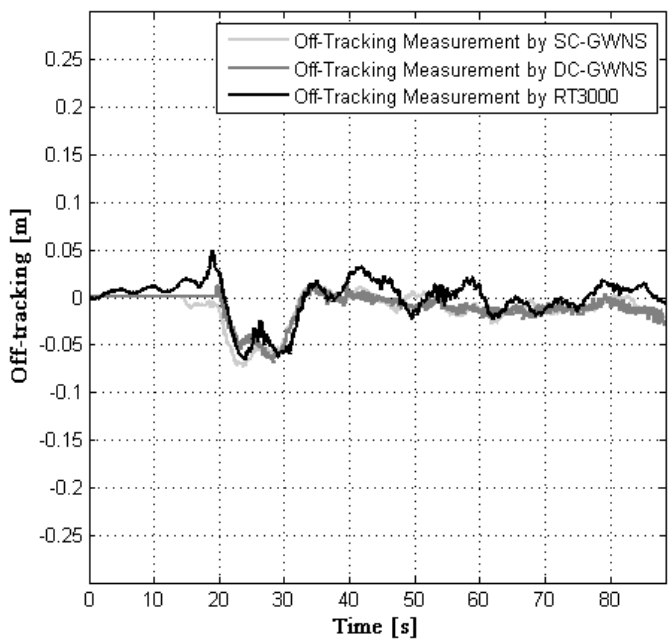

Fig. 7. Open-loop testing results for the straight-line manoeuvre

between the lead and follow points in the simulation (11.5m). The uncertainty of the integrated positions calculated by the SC-GWNS is therefore smaller.

Closed-loop testing results for the straight-line are shown in
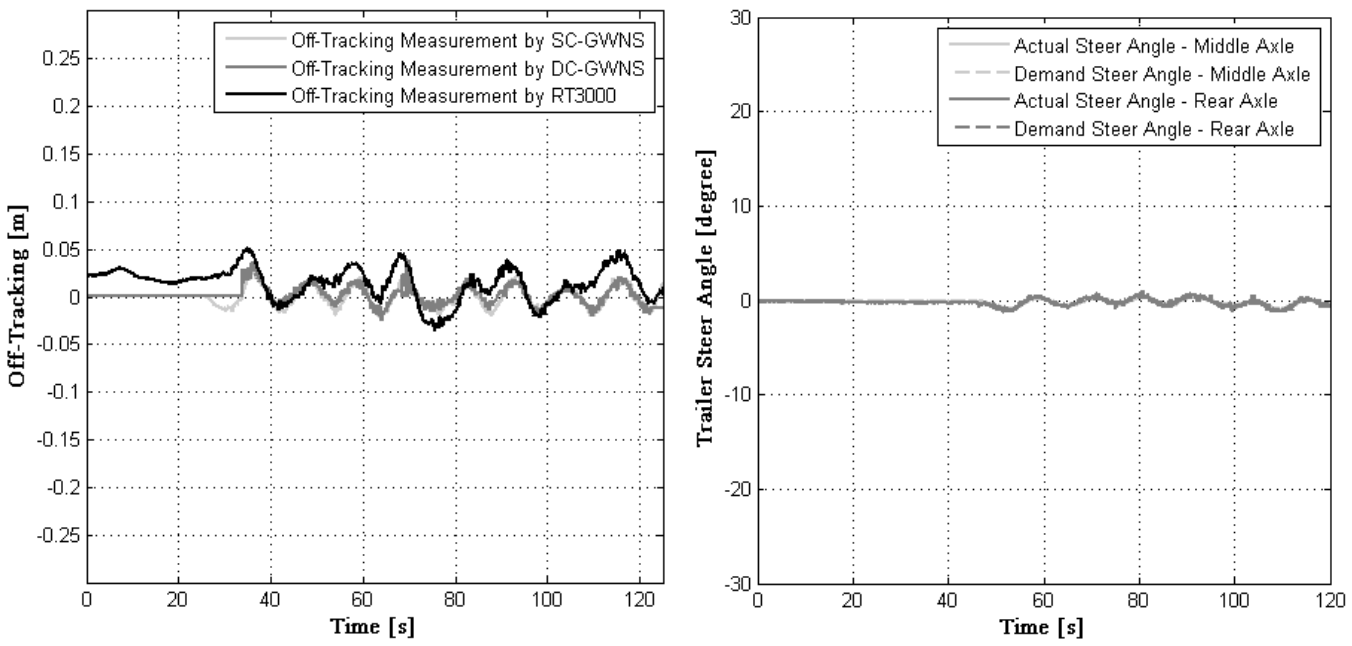

(a), (b) off-tracking distances and trailer steer angles - trailer steered based on the signals from the SC-GWNS
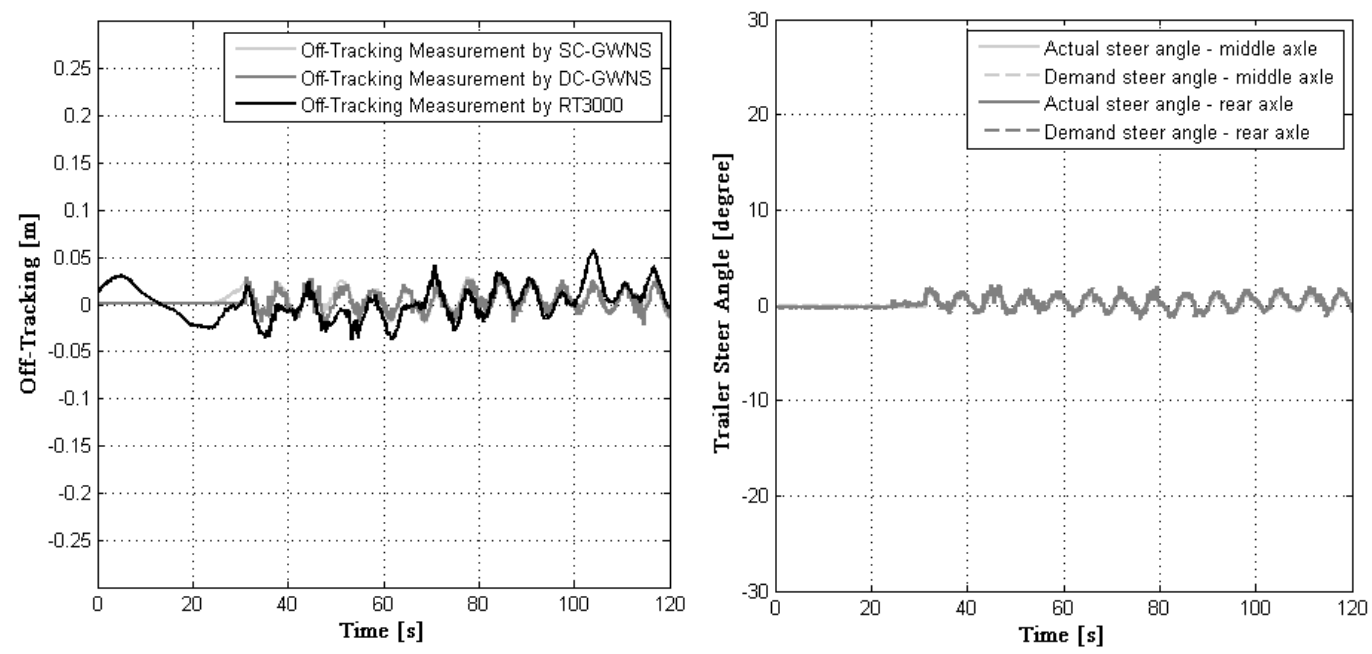

(c), (d) off-tracking distances and trailer steer angles - trailer axles steered based on the signals from the DC-GWNS

Fig. 8. Closed-loop testing results for the straight-line 
Fig. 8. Path-following performance based on the SC-GWNS is shown in Fig. 8 (a) with trailer steer angles shown in Fig. 8 (b). Path errors based on the DC-GWNS are shown in Fig. 8 (c). It can be seen from the figures that off-tracking distances at the rear camera with regard to the front camera were controlled within $\pm 0.03 \mathrm{~m}$ for the straight-line tests with the DC-GWNS. This performance is similar to the open-loop tests with unsteered trailer axles. The off-tracking signals measured by the 3 navigation systems agreed with each other well.

For the straight-line tests, the actual steer angles (solid lines in Figs 8 (b) and (d)) tracked the demand steer angles (dashed lines) very well. The steer angles for the middle and rear axles were both around $0^{\circ}$ with some oscillations less than $2^{\circ}$, at the same frequencies as the off-tracking errors in Figs 8 (a) and (c).

There is a clear low frequency oscillation in each case: with a 20 s period (corresponding to a wavelength of approximate $30 \mathrm{~m}$ ) for the SC-GWNS in Fig. 8 (a) and approximately 10s $(15 \mathrm{~m})$ for the DC-GWNS in Fig. 8 (c).The cause of this oscillation is not completely clear. However, the characteristics are similar to those described by Rimmer in her research on autonomous reversing of articulated vehicles [15]. Rimmer's tests were also conducted at Bourn field at low speeds and the
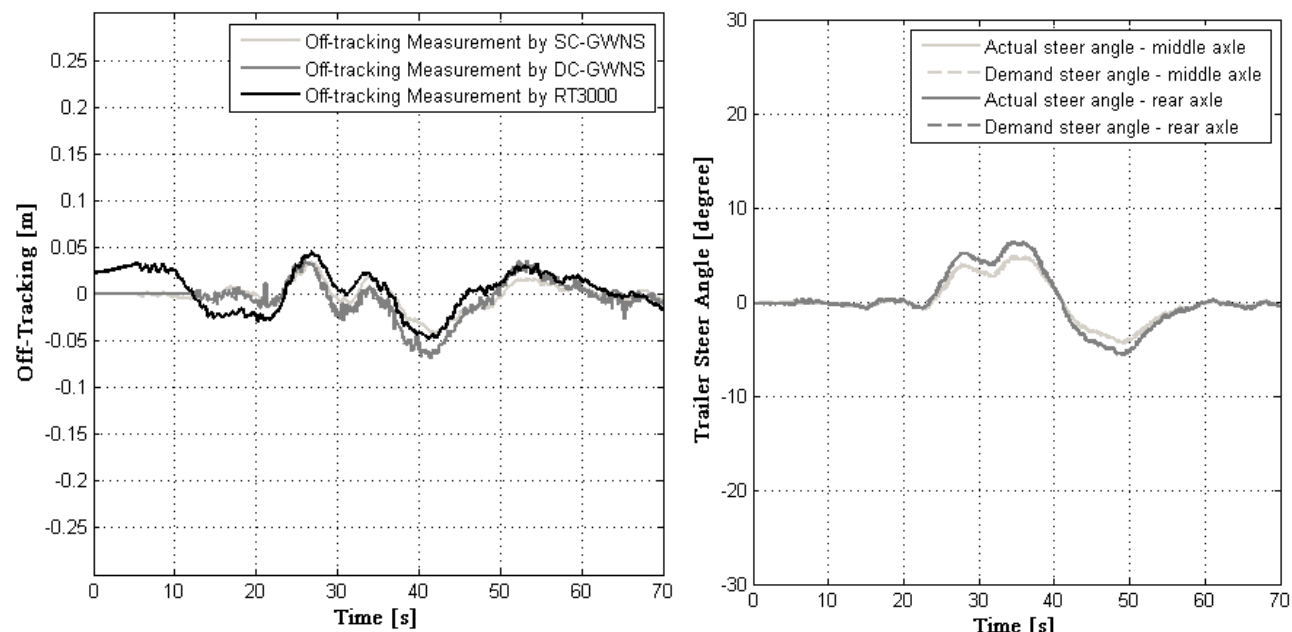

(a), (b) off-tracking distances and trailer steer angles - trailer steered based on the signals from the SC-GWNS
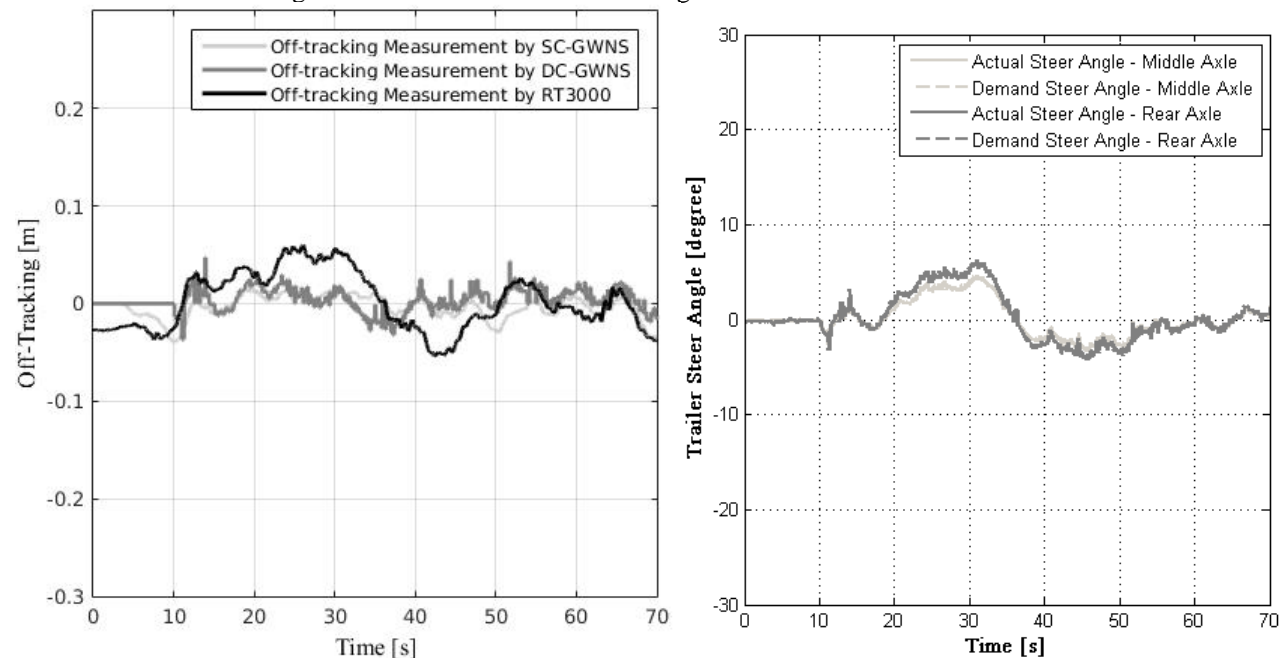

(c), (d) off-tracking distances and trailer steer angles - trailer steered based on the signals from the DC-GWNS

Fig. 10. Closed-loop testing results for the lane change. 
the steering of the front axle.)

In Rimmer's case, the oscillations were found to be caused by the relatively large cross-slope roughness of the road surface introducing a lateral tyre force disturbance to the vehicle. This interacted with low damping in the controller to create the oscillations. Rimmer found that the oscillations could be reduced slightly by retuning the controller gains. Although attempts were made to reduce the oscillations in the GWNS through controller tuning, time constraints prevented a complete solution to the problem. Consequently, some low frequency oscillations are seen on all of the closed loop test results.

\section{2) Lane-change}

Open-loop testing results for the lane-change are shown in Fig. 9. It can be seen from the figure that the off-tracking distance was within $\pm 0.02 \mathrm{~m}$ for the straight-line, and then varied between $\pm 0.15 \mathrm{~m}$ during the lane change. Off-tracking distance signals from the two GWNSs agree with each other very well, and exhibit errors smaller than $0.05 \mathrm{~m}$ compared with the RT3000 measurements. However, between points P and Q the level of off-tracking exceeded $0.25 \mathrm{~m}$, the maximum amount that can be measured by the DC-GWNS, because the overlap distance between the images became too small.

Closed-loop testing results for the lane-change are shown in Fig. 10. Path-following performance based on the SC-GWNS and the DC-GWNS are shown in Figs. 10 (a) and (c) respectively. Off-tracking measurements from the RT3000 are presented as black lines in the figures. It can be seen from the figures that the controllers performed accurate path-tracking within $\pm 0.05 \mathrm{~m}$ of the RT3000 measurements. The off-tracking distance was reduced by up to $0.3 \mathrm{~m}$ compared with the open-loop tests (Fig. 9). Some low frequency oscillations are visible on these results, although the amplitudes are less than for the straight-line tests in fig. 8 .

Trailer steer angles are provided in Figs. 10 (b) and (d). For the lane change, the front axle of the tandem group steered $\pm 4^{\circ}$ while the rear axle was steered up to $\pm 5^{\circ}$. The actual steer angles followed the demand steer angles (dashed lines) with small errors and delays.

\section{3) $90^{\circ}$ corner}

Open-loop testing results for the $90^{\circ}$-corner are shown in Fig. 11. It can be seen from the figure that the off-tracking distance increased to $1.8 \mathrm{~m}$ during the corner. The SC-GWNS signals agreed well with the RT3000 signals, showing the maximum measurement errors of less than $0.05 \mathrm{~m}$ throughout the manoeuvre. The DC-GWNS got 'lost' after approximately $28 \mathrm{~s}$ (point P) when off-tracking distance exceeded $0.25 \mathrm{~m}$ but 'locked-in' again at approximately 65s (point Q) when off-tracking dropped below $0.25 \mathrm{~m}$.

Closed-loop testing results for the $90^{\circ}$ corner are shown in Fig. 12. Path-following performance based on the SC-GWNS (light grey) and the DC-GWNS (dark grey) are shown in Figs. 12 (a) and (c) respectively. In Fig. 12 (a) the off-tracking distance exceeded $0.05 \mathrm{~m}$ and $-0.1 \mathrm{~m}$ when the test vehicle entered and exited the corner, respectively. A more accurate

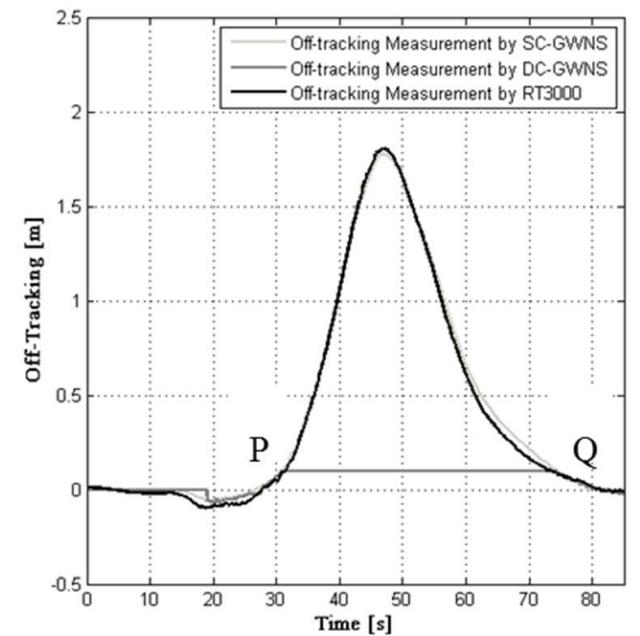

Fig. 11. Open-loop testing results for the $90^{\circ}$ corner

tracking response is exhibited by the DC-GWNS in Fig. 12 (c), which showed accurate path-following within $\pm 0.05 \mathrm{~m}$ for the entire manoeuvre. Compared to the performance of the same manoeuvre in open-loop testing (Fig. 11), off-tracking was considerably reduced by over $1.8 \mathrm{~m}$.

Trailer steer angles are provided in Figs. 12 (b) and (d). For the $90^{\circ}$ corner, demand and actual steer angles for the middle axle increased from $0^{\circ}$ for the straight-line to $20^{\circ}$ during the corner. The counterparts for the rear axle varied between $0^{\circ}$ and $25^{\circ}$. The demanded and actual steer angles agree closely in each case, indicating good co ntrol performance. The different steer angle demands for the two axles are due to the vehicle geometry in the sharp corner which required large but different steer angles from the two axles to counteract the cut-in without sideslip of the tyres.

\section{CONCLUSIONS AND FUTURE WORK}

A trailer-based path-following controller was developed based on Cheng's work. Two different ground-watching navigation systems (GWNSs) were employed to provide navigation data for the controllers. Theoretical performance of steering control based on GWNSs was investigated in closed-loop simulations. The dual-camera system (DC-GWNS) was found to outperform the single-camera system (SC-GWNS) in measurement accuracy but has constraints on the measurement range.

Theoretical performance of steering control based on GWNSs was investigated in closed-loop simulation for the $450^{\circ} \mathrm{UK}$ standard roundabout manoeuvre. The DC-GWNS were found to outperform the SC-GWNS in measurement accuracy but have constraints on the measurement range.

Two ground-watching navigation systems (GWNS) were developed and implemented on the CVDC active steering test vehicle. A multi-level control scheme was implemented on the test vehicle to perform path-following using measurement signals from the GWNSs.

Vehicle testing was performed at $5 \mathrm{~km} / \mathrm{h}$ for 3 manoeuvres: a straight-line, a lane-change and a $90^{\circ}$ corner. Open-loop tests 

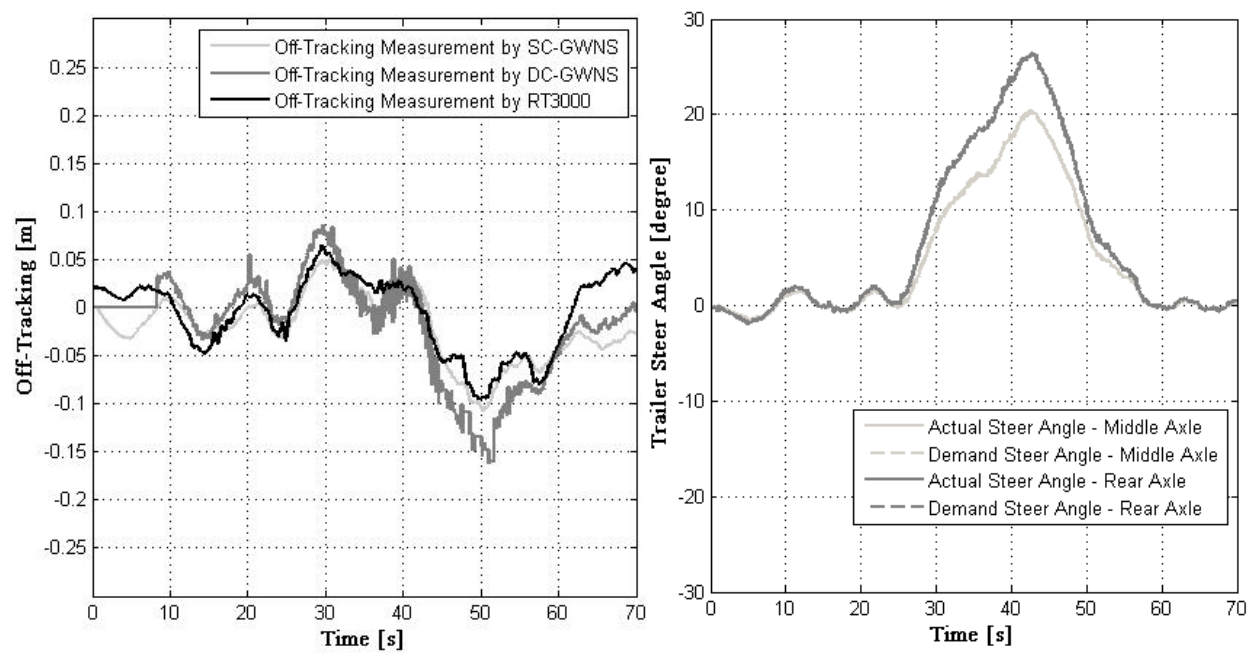

(a), (b) off-tracking distances and trailer steer angles - trailer steered based on the signals from the SC-GWNS
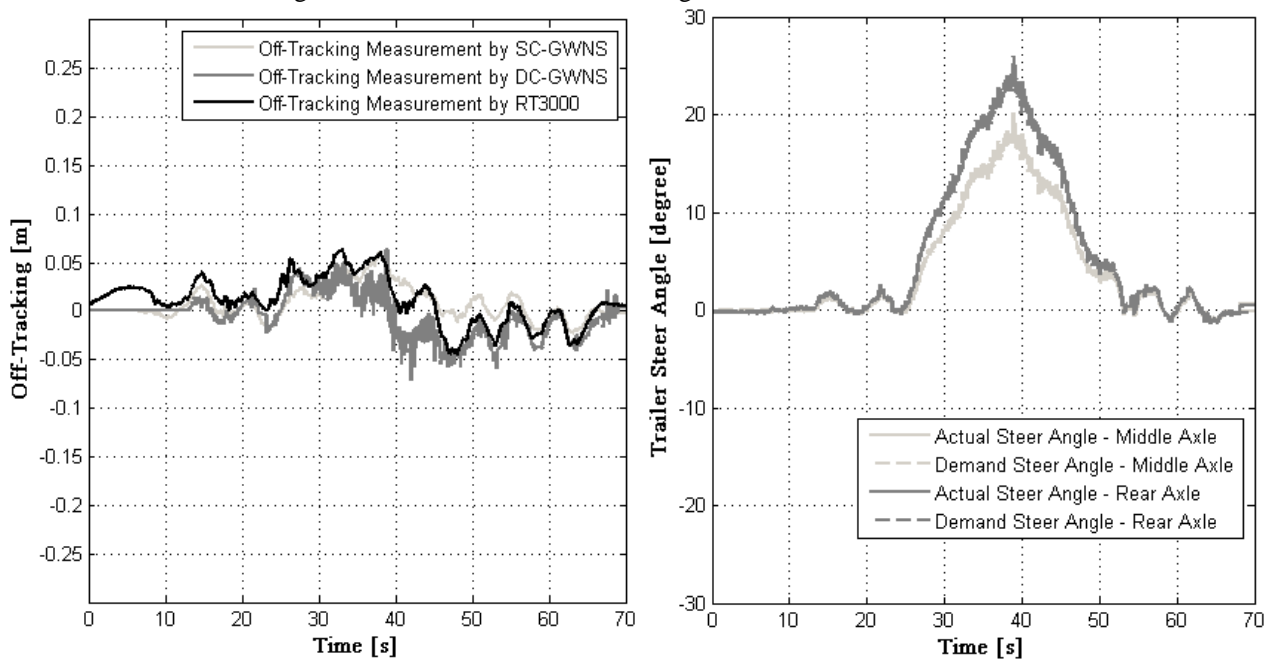

(c), (d) off-tracking distances and trailer steer angles - trailer steered based on the signals from the DC-GWNS

Fig. 12. Closed-loop testing results for the $90^{\circ}$ corner

were conducted with trailer axles locked to the centre and closed-loop tests were performed with trailer axles operated in 'path-following' mode. Care was taken to zero the bias in yaw rate at the start of each test.

The closed-loop vehicle testing showed that the active steering controller with input signals from the SC-GWNS and the DC-GWNS performed accurate path-following at low speeds. Off-tracking distance at the rear camera with regard to the front camera was controlled below $0.1 \mathrm{~m}$ for the 3 manoeuvres. Small, low frequency oscillations, probably due to lateral tyre force disturbance caused by the roughness of the test ground, were present on the test results.

The concept of GWNS-based trailer steering control was proved to work well for a series of low speed manoeuvres on asphalt pavement. The important next experimental step will be to test the vehicle on slippery roads with camber and grade. This will determine the effectiveness of the system for the conditions that it was designed to handle.

Although the ground-watching strategy has been shown to work accurately under ideal conditions, there are likely to be practical difficulties associated with keeping the cameras clean and operating on soft or muddy ground. An alternative would be to use a similar steering control strategy but with visual information from the surrounding scene, rather than the road, to measure off-tracking. This is the subject of current research.

\section{REFERENCES}

1. Jujnovich, B.A., Active Steering of Articulated Vehicles, 2005, University of Cambridge: Cambridge, UK.

2. Woodrooffe, J., Economic Efficiency of Long Combination Transport Vehicles in Alberta, 2001, Woodrooffe \& Associates: Ottawa, Canada.

3. Miao, Q. and D. Cebon, Effects of Operating Off-Highway on the Tracking Performance of a Path-Following Steering System, in FISITA 2014 World Automotive Congress2014: Maarstricht, Netherland.

4. Jones, P. and A. Wright, Command Steer Trailer Steering, 1995, Aston University: Birmingham, UK.

5. Miao, Q., Vision - based Path - Following Control of Articulated Vehicles, in Department of Engineering 2015, University of Cambridge: Cambridge, UK.

6. Hofmann-Wellenhof, B., et al., Global Positioning System: Theory and practice. Revised Edition, 2001: Springer.

7. Cheng, C., Enhancing Safety of Actively-Steered Articulated Vehicles, 2009, University of Cambridge: Cambridge, UK

8. Åström, K.J. and R.M. Murray, Feedback Systems2008: Princeton 
University Press.

9. Ziegler, J.G. and N.B. Nichols. Optimum Settings for Automatic Controllers. in Trans. ASME. 1942.

10. Anon, RT3000 Inertial and GPS Measurement System: User Manual2004, Oxford, UK: Oxford Technical Solutions.

11. Rimmer, A., Autonomous Reversing of Multiply-Articulated Heavy Vehicles, in Engineering Department, 2015, University of Cambridge: Cambridge, UK.

12. Roebuck, R.L., et al., Implementation of Trailer Steering Control on a Multi-Unit Articulated Vehicle at High Speeds. ASME Journal of Dynamic Systems, Measurement and Control, 2014. Vol. 136(No. 2).

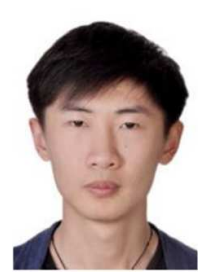

Qiheng Miao received the B.Eng. degree in automotive engineering from Jilin University, China, in 2011. He is currently pursuing the Ph.D. degree with Professor David Cebon in the Department of Engineering at Cambridge University. His research interests include: image processing, vehicle dynamics and active steering control of articulated vehicles.

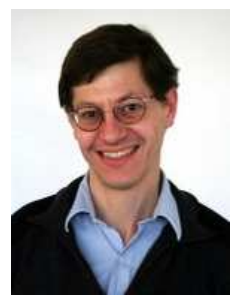

David Cebon received BE in mechanical engineering from University of Melbourne, Australia in 1980 and Ph.D. degree in engineering from University of Cambridge, UK, in 1985.

He has been employed by the University of Cambridge since 1985, first as a lecturer and more recently as professor of mechanical engineering. His research covers the mechanical, civil, and materials aspects of road transport engineering. He has authored or co-authored more than 150 papers on dynamic loads of heavy vehicles, road and bridge response and damage, advanced suspension design for heavy vehicles, heavy vehicle safety, mobility and fuel consumption and the micromechanics of asphalt deformation and fracture. 